\title{
Mapping atmospheric depositions of cadmium and lead in Germany based on EMEP deposition data and the European Moss Survey 2005
}

\section{Kartierung atmosphärischer Depositionen von Blei und Cadmium in Deutschland mit Daten aus dem EMEP-Messnetz und dem europäischen Moos- Monitoring 2005}

Winfried Schröder ${ }^{1 \dagger}$, Marcel Holy ${ }^{1 \dagger}$, Roland Pesch*1+, Harald Zechmeister ${ }^{2+}$ Harry Harmens ${ }^{3}$ and llia llyin ${ }^{4}$

\begin{abstract}
Background Every five years since 1990, the European Heavy Metals in Mosses Survey provided data on atmospheric heavy metal bioaccumulations in mosses throughout Europe at a high spatial resolution. The moss data show the effectiveness of air quality control policies: for Germany the metal bioaccumulations decreased between 1990 and 2000, whilst they increased from 2000 to 2005. This investigation is intended to show how the moss data could be used to map atmospheric depositions of $\mathrm{Cd}$ and $\mathrm{Pb}$, which later on might serve for the calculation of Critical Loads Exceedances. In addition, we compared how much heavy metal concentrations in mosses in Germany deviate from background data observed in Greenland.

Methods Mapping heavy metals with a high spatial resolution for the German territory was conducted according to the following methodology: EMEP deposition maps (50 km by $50 \mathrm{~km}$ spatial resolution) were intersected within a GIS with Kriging maps on $\mathrm{Cd}$ and $\mathrm{Pb}$ accumulations in mosses (EMEP (European Monitoring and Evaluation Programme) is a scientifically based and politically driven programme under the Convention on Long-range Transboundary Air Pollution for international co-operation to solve transboundary air pollution problems). Subsequently, the statistical relations between the EMEP modelled depositions and the bioaccumulations in mosses were quantified by using regression analysis. The regression functions were used to transform the moss concentration maps into deposition maps. The resulting maps on $\mathrm{Cd}$ and $\mathrm{Pb}$ depositions have a spatial resolution of $5 \mathrm{~km}$ by $5 \mathrm{~km}$ and were added to the respective map on the residuals of the regression functions (Regression Kriging). Finally, the territory of Germany was extracted from the European maps on $\mathrm{Cd}$ and $\mathrm{Pb}$ depositions and the legends were adjusted accordingly in terms of $\mathrm{n}$ standard deviations from the German mean value. The concentrations of Al, As, Cd, Cr, Cu, Mo, Pb, Sb, Sn, and Zn in the mosses sampled in 1990, 1995, 2000 and 2005 in Germany were compared with background values derived from mosses sampled in north-eastern Greenland (Zackenberg Background Values). The differences between heavy metal concentrations in mosses in Germany and Greenland were calculated for the 16 federal states of Germany and mapped for $\mathrm{Pb}$
\end{abstract}

\footnotetext{
†These authors contributed equally to this work

*Correspondence: rpesch@iuw.uni-vechta.de

${ }^{1}$ Chair of Landscape Ecology, University of Vechta, P.O.B. 1553, 49364 Vechta,

Germany
}

Full list of author information is available at the end of the article

\section{SpringerOpen ${ }^{\circ}$}

(c) 2011 Schröder et al; licensee Springer. This is an open access article distributed under the terms of the Creative Commons Attribution License (http://creativecommons.org/licenses/by/2.0), which permits unrestricted use, distribution, and reproduction in any medium, provided the original work is properly cited. 
Results and discussion The regression models corroborate that the $\mathrm{Cd}$ concentration in mosses is correlated with the EMEP modelled total Cd deposition across Europe (regression coefficient according to Pearson, $r_{p}=0.67$; regression coefficient according to Spearman, $r_{s}=0.69$ ). The coefficient of determination is $r^{2}=0.44$. The same is true for $\mathrm{Pb}$ with $r_{p}=0.76$ and $r_{s}=0.77$ and $r^{2}=0.58$. Based on the regression models and the respective residuals, maps on the total deposition of $\mathrm{Cd}$ and $\mathrm{Pb}$ were calculated for the year 2005. The German mean value of total $\mathrm{Cd}$ and $\mathrm{Pb}$ deposition was $0.342 \mathrm{~g} / \mathrm{ha} / \mathrm{a}$ (standard deviation $0.08 \mathrm{~g} / \mathrm{ha} / \mathrm{a}$ and $8.6 \mathrm{~g} / \mathrm{ha} / \mathrm{a}$ (standard deviation $2.1 \mathrm{~g} / \mathrm{ha} / \mathrm{a}$ ) respectively. The maps depict the spatial patterns of the total $\mathrm{Cd}$ and $\mathrm{Pb}$ deposition in terms of $\mathrm{n}$ standard deviations from the respective German wide mean value. The spatial resolution of the maps is $5 \mathrm{~km} \times 5 \mathrm{~km}$ and reflects the mesh size of the moss monitoring net. Even today, the bioaccumulation of several metals in Germany still exceeds the background values observed in Greenland. This is true especially for $\mathrm{Cd}, \mathrm{Cr}, \mathrm{Cu}, \mathrm{Mo}, \mathrm{Pb}, \mathrm{Sb}, \mathrm{Sn}$ and $\mathrm{Zn}$. Comparing the results of this investigation with those from other methods it can be concluded that the mean values calculated for the total $\mathrm{Cd}$ and Pb deposition for Germany differ from such assessed by deposition measurements and models. The latter are used to calculate Critical Loads Exceedances, which complement the ecotoxicological endpoint 'accumulation'. The deposition measurements in Germany are mainly based on monitoring systems conducted by the federal states. When trying to use deposition measurements from the ICP Forests level II programme for the validation of the EMEP deposition modelling, problems arose due to a lack of methodical harmonization and the quality of the depositions measurements. That is why in this investigation the quality controlled and spatially high resolved moss data were used to empirically validate EMEP modelled deposition maps.

Conclusions In Germany, the moss measurement data provide a valuable tool at a high spatial resolution for the validation of modelling and mapping of atmospheric heavy metal deposition and should as such be used for this purpose. The comparison of the metal concentrations in mosses in Germany with the values found in Greenland indicate that atmospheric deposition of heavy metals in Germany is still considerably higher than the natural background deposition.

Keywords EMEP deposition network and modelling; European Moss Survey as part of UNECE ICP Vegetation; Mapping atmospheric depositions of $\mathrm{Cd}$ and Pb; Zackenberg Background Values

\section{Zusammenfassung}

Hintergrund Das Moos-Monitoring liefert seit 1990 alle fünf Jahre flächendeckende, qualitätskontrollierte Daten über die Bioakkumulation von Schwermetallen und Stickstoff (seit 2005) in Europa. Die Monitoring-Daten belegen eindrücklich, wie sich Luftreinhaltepolitik auf die Anreicherung atmosphärischer Depositionen auswirkt. Das MoosMonitoring reagiert auch sensibel auf eine Trendumkehr: Nachdem die Metallanreicherungen von 1990 bis 2000 deutlich sanken, stiegen die Gehalte einiger Metalle bis 2005 wieder an. Depositionsmessungen konnten diesen Trend, der auch in anderen europäischen Staaten nur durch das Moos-Monitoring entdeckt wurde, nicht detektieren. Ziel dieser Untersuchungen ist es zu zeigen, dass die Daten des Moos-Monitoring nicht nur zur Kartierung der Bioakkumulation atmosphärischer Depositionen verwendet werden können, sondern auch zur räumlich hochauflösenden Kartierung von Schwermetall-Gesamtdepositionen als Grundlage für die Berechnung von Critical Loads Exceedances. Wie weit die Bioakkumulation von Metallen in Deutschland von einem quasi-natürlichen Zustand abweicht, soll anhand geeigneter Referenzwerte bestimmt werden.

Methoden Die räumlich hoch auflösende Kartierung atmosphärischer Schwermetall-Gesamtdepositionen für das Gebiet der Bundesrepublik Deutschland basiert auf folgenden methodischen Fundamenten: Die auf der Grundlage europaweit harmonisierter Messungen atmosphärischer Depositionen von Cd und Pb (Jahr 2005) an 70 Orten Europas berechneten flächendeckenden EMEP-Depositionskarten (räumliche Auflösung $50 \mathrm{~km}$ mal 50 km) wurden mit geostatistich validen Kriging-Karten über die Anreicherung von $\mathrm{Cd}$ und $\mathrm{Pb}$ in europaweit gesammelten Moosen in einem geografischen Informationssystem (GIS) verschnitten (EMEP (European Monitoring and Evaluation Programme) is a scientifically based and politically driven programme under the Convention on Long-range Transboundary Air Pollution for international co-operation to solve transboundary air pollution problems). Sodann wurden die statistischen Beziehungen zwischen den im EMEP-Depositionsnetz und im ICP Vegetation Moosmessnetz erhobenen $\mathrm{Cd}$ - und Pb-Depositionen sowie den Anreicherungen dieser beiden Metalle regressionsanalytisch quantifiziert. Mit den Regressionsfunktionen wurden anschließend die hoch aufgelösten Kriging-Karten der Cd- bzw. $\mathrm{Pb}$-Gehalte der Moose in räumlich hoch aufgelöste Depositionskarten umgerechnet. Die dadurch entstandenen $\mathrm{Cd}$ - 
und Pb-Depositionskarten (räumliche Auflösung $5 \mathrm{~km} \times 5 \mathrm{~km}$ ) wurden schließlich noch dadurch ergänzt, dass für die Residuen der beiden Regressionsmodelle Kriging-Karten erzeugt und diese mit den $\mathrm{Cd}$ - und Pb-Regressionskarten verrechnet wurden (Regression-Kriging). Sodann wurde das Gebiet der Bundesrepublik aus den Europakarten der $\mathrm{Cd}$ - und Pb-Deposition ausgestanzt und die Legendierungen an den Wertebereich im Bereich der Bundesrepublik Deutschland als n-fache Abweichungen vom Bundesdurchschnitt angepasst. Schließlich wurden Karten berechnet, in denen für jedes Bundesland die Pb-Depositionen als Abweichungen von dem jeweiligen Grönländischen Hintergrundwert dargestellt sind.

Ergebnisse und Diskussion Die Regressionsmodelle zeigen, dass die Cd-Anreicherungen in den Moosen mit der Cd-Gesamtdeposition europaweit hoch korreliert sind (Regressionskoeffizient nach Pearson, $r_{p}=0.67$; Regressionskoeffizient nach Spearman, $\left.r_{s}=0.69\right)$. Das Bestimmtheitsmaß des Regressionsmodells beträgt $r^{2}=0,44$. Die Pb-Akkumulationen in den Moosen korrelieren mit der Pb-Gesamtdeposition deutlich $\left(r_{p}=0.76, r_{s}=0.77\right)$. Das Bestimmtheitsmaß des Regressionsmodells beträgt $r^{2}=0,58$. Auf dieser Grundlage wurde je eine Karte der $\mathrm{Cd}$ - und Pb-Gesamtdeposition berechnet, die jeweils mit einer Kriging-Karte der Residuen des Regressionsmodells zu je einer Ergebniskarte zusammengefasst wurde. Demnach betrug die Cd-Gesamtdeposition im deutschlandweiten Mittel 0,34 g / ha / a (Standardabweichung 0,08 g / ha / a). Die bundesweit durchschnittliche Pb-Gesamtdeposition betrug 8,6 g / ha / a (Standardabweichung 2,1 g / ha / a). Die Karten zeigen die räumliche Differenzierung der Cd- und $\mathrm{Pb}-G e s a m t d e p o s i t i o n$ als Vielfache der jeweiligen Standardabweichung. Die dabei gewählte räumliche Auflösung orientiert sich an der Standardabweichung der Distanzen zwischen je zwei Beprobungspunkten in Deutschland und beträgt dementsprechend $5 \mathrm{~km}$ mal $5 \mathrm{~km}$. Schließlich wurden die Gehalte von Al, As, Cd, Cr, Cu, Mo, Pb, Sb, Sn und Zn der 1990, 1995, 2000 und 2005 in Deutschland gesammelten Moosen auf europäische Hintergrundwerte bezogen, die in Moosen Nordost Grönlands ermittelt wurden. Die Abweichungen zwischen den Pb-Anreicherungen in Deutschland und in Grönland wurden - nach Bundesländern differenziert - kartiert. Es zeigt sich, dass noch heute deutliche Unterschiede in der Anreicherung atmosphärisch deponierter Metalle zwischen Deutschland und Grönland bestehen, insbesondere bei $\mathrm{Cd}, \mathrm{Cr}, \mathrm{Cu}, \mathrm{Mo}, \mathrm{Pb}, \mathrm{Sb}, \mathrm{Sn}$, and $\mathrm{Zn}$. Vergleicht man die Ergebnisse des in dieser Arbeit vorgestellten Ansatzes der Depositionskartierung mit Ergebnissen aus anderen Verfahren, so zeigen sich Unterschiede. Ergebnisse aus Depositionsmessungen und -modellierungen werden u.a. dafür verwendet, Critical Loads zu berechnen und zu kartieren und damit über den Wirkungsaspekt der Anreicherung hinauszugehen. Die Depositions-modellierungen in Deutschland basieren u.a. auf Depositionsmessungen der Bundesländer, die methodisch weiter harmonisiert werden sollten. Die auf die Depositionsdaten zurückgreifenden Modellierungen sollten transparenter gestaltet werden. Bei dem Versuch, für die europaweite Depositionsmodellierung (EMEP) Depositionsdaten aus dem UNECE ICP Forests-Messnetz zur Validierung zu nutzen, gab es Probleme unzureichender Datenharmonisierung. Deshalb wurde in dieser Arbeit angestrebt, die in hoher räumlicher Dichte vorliegenden qualitätskontrollierten Daten des im UNECE ICP Vegetation durchgeführten Moos-Monitoring dafür zu verwenden, die räumliche Differenzierung der Depositionskarten empirisch abzusichern. Fachlich unbefriedigend bleibt, dass bei der Depositionsmodellierung in Deutschland anders als auf internationaler Ebene (EMEP / ICP Vegetation MoosMonitoring) eine Verknüpfung von Depositionsdaten und Akkumulationsdaten aus dem Moos-Monitoring bislang nicht zustande kam. Der Abgleich der Bioakkumulation in Deutschland mit den Zackenberg Hintergrundwerten zeigt, wie weit die atmosphärischen Einträge in Deutschland von einem quasinatürlichen Zustand entfernt sind.

Schlagwörter EMEP Depositionsmessnetz und -modellierung; Europäisches Moos-Monitoring im UNECE ICP Vegetation; Kartierung atmosphärischer Cd- und Pb-Depositionen in Deutschland; Zackenberg Hintergrundwerte

\section{Hintergrund}

Die Messung von Stoffen, die aus der Atmosphäre an der Erdoberfläche abgeschieden werden (atmosphärische Depositionen), ist eine wesentliche Grundlage für die Beschreibung und Bewertung der Umweltqualität. Ergebnisse aus Depositionsmessungen und -modellierungen werden u.a. dafür verwendet, Critical Loads zu berechnen und $\mathrm{zu}$ kartieren und damit über den Wirkungsaspekt der Anreicherung hinauszugehen [1,2]. Die atmosphärischen Depositionen bilden für Organismen deren externe stoffliche Exposition. Ihr kann sich die Anreicherung der deponierten Stoffe an der äußeren Hülle des Lebewesen und der Stofftransport in innere Organe anschließen [3,4]. Das seit 1990 alle fünf Jahre europaweit durchgeführte Moos-Monitoring erfasst die Anreicherung und somit die potenzielle Bioverfügbarkeit von Schwermetallen [5,6]. Zwar ermöglicht es das dabei angewendete experimentelle Design nicht, zwischen externer und interner Bioverfügbarkeit zu differenzieren, doch ist bekannt, dass die externe Bioverfügbarkeit von 
Metallen ihre interne Verteilung und Wirkung beeinflussen kann [7]. Das bedeutet, dass Daten selbst dann, wenn sie nur die externe Bioverfügbarkeit der Schwermetalle erfassen sollten, toxikologisch bedeutsam sind [8]. Die Daten aus dem Moos-Monitoring erfassen also zumindest die externe Schwermetall-Bioverfügbarkeit und ergänzen die Daten aus Depositionsmessnetzen, welche die Stoffkonzentration im Expositionsmedium Atmosphäre und somit einen Teil der externen Stoffexposition von Landlebewesen kennzeichnen. Doch nicht nur geochemische Prozesse an der Grenzfläche zwischen Atmosphäre und Lebewesen bestimmen die Bioverfügbarkeit, sondern auch biochemische Prozesse an der biologischen Oberfläche sowie jenseits davon im Lebewesen selbst, einschließlich toxischer Wirkungen [9]. Die Verfügbarkeit von Metallen an der äußeren Hülle von Lebewesen ist also grundsätzlich mit einem Toxizitätspotenzial verknüpft.

Die Verbesserung der (öko)toxikologischen Bewertungsgrundlagen für Metalle ist Gegenstand der REACHVerordnung und der Europäischen Wasserrahmenrichtlinie [10] (REACH, Registration, Evaluation, Authorisation and Restriction of Chemicals, Verordnung (EG) Nr. 1907/2006). Ihre Umsetzung erfordert zuverlässige, räumlich angemessen differenzierte Umweltdaten [11]. Dazu gehören auch Depositionsdaten. Diese erhalten einen Wirkungsbezug, wenn sie bei der Berechnung von Critical Loads (CL) [12,13] als Predicted Environmental Concentration (PEC) mit Predicted No Effect Concentrations (PNEC) von Ökosystemen verknüpft werden [14]. Die Aussagekraft von CL hängt von der Qualität des Modells. d.h. von seiner intrinsischen (Un)Sicherheit sowie von der Qualität der Eingangsdaten, insbesondere die von Emissionsdaten und die im Forstmonitoring erhobenen Depositionsdaten ab $[15,16]$. Besondere Bedeutung kommt auch der räumlichen Auflösung der Depositionsdaten zu [13,17].

Um die räumliche Validität und damit die Aussagekraft von Critical Loads Exceedance-Karten empirisch zu untermauern, liegt es nahe, die statistischen Beziehungen zwischen Depositionsdaten und Daten aus dem räumlich recht dichten Messnetz des Europäischen MoosMonitoring regressionanalytisch zu quantifizieren und die Regressionsmodelle auf die Messpunkte des Moosmessnetzes oder die daraus abgeleiteten flächendeckenden und räumlich hoch aufgelösten Karten der Schwermetallakkumulation in Moosen anzuwenden. Ziel dieses Artikels ist es, die dazu angewendete Methodik und die erzielten Ergebnisse für die Deposition der Schwermetalle Blei (Pb) und Cadmium (Cd) im Jahr 2005 für das Gebiet der Bundesrepublik Deutschland aufzuzeigen. Ferner zielt diese Arbeit darauf ab, zu bestimmen, wie weit die Bioakkumulation von Metallen in Deutschland von einem quasi-natürlichen Zustand abweicht.

\section{Methoden \\ Moos-Monitoring}

Da Moose über mehrere Jahre die Anreicherung von Metallen ohne physiologische Beeinträchtigung tolerieren, sind sie besonders geeignet für das Monitoring der Bioakkumulation atmosphärischer Depositionen. Dies gilt nicht nur für immittentenbezogene Untersuchungen auf lokaler und regionaler Ebene. Besonders für die Erfassung großräumiger Trends ist das Moosmonitoring geeignet [18] und wird deshalb in Europa seit 1990 alle fünf Jahre in mindestens 21 Ländern durchgeführt [5,6]. Ziel ist es dabei, die großräumigen Muster und zeitlichen Entwicklungen der auf Langstreckentransport und atmosphärische Deposition zurückzuführende Metallbioakkumulation Staaten übergreifend $\mathrm{zu}$ kartieren. Dabei geht es in erster Linie darum, sogenannte Hintergrundwerte zu erfassen und nicht darum, emittentenbezogene Aussagen zu gewinnen. Von potenziellen Emissionsquellen sind in der anzuwendenden Richtlinie vorgegebene räumliche Abstände einzuhalten, und an Orten mit sichtbaren technischen Emissionsquellen in ihrer Umgebung werden keine Moosproben entnommen [19].

Diese Richtlinie gilt nicht nur für die Entnahme, sondern auch für die chemischen Analysen der Moosproben. Europaweit sollten auf $1000 \mathrm{~km}^{2}$ mindestens 1,5 Moosentnahmestellen entfallen. Das bedeutet je nach Anzahl der an einer Messkampagne teilnehmenden Staaten, dass an bis zu rund 7000 Orten in Europa Moosproben entnommen und analysiert wurden. In Deutschland führten Bund und Länder das MoosMonitoring in den Jahren 1990, 1995, 2000 und 2005 gemeinsam durch. Im Jahr 2009 hat sich der Bund aus dem Moos-Monitoring zurückgezogen. Die räumliche Dichte des Moosmessnetzes betrug 1,7 (1990), 2,9 (1995, 2000) und 2,0 (2005) Standorte pro $1000 \mathrm{~km}^{2}$. Die Messnetzausdünnung beim deutschen Monitoring in 2005 erfolgte ohne Einbußen der geostatistischen Validität und Landschaftsrepräsentanz des Messnetzes [20].

Die Qualität der Messungen und Probenentnahmen wurde - beginnend mit der Planung und der Schulung der Probenentnehmer und mit dem Datenmanagement endend [21] - in mehreren Schritten gesichert und dokumentiert [5,6,14,22]: Die analytischen Kontrollen erfolgten wie in anderen Teilnehmerstaaten durch die Messung der Referenzmaterialien M2 (hohe Metallkonzentrationen) und M3 (regionale Hintergrundwerte im Sinne von [23] in jeder Messserie sowie den Austausch und die Messung von Moosproben, die auf beiden Seiten der Grenzen benachbarter Staaten gesammelt wurden. Die Daten wurden von den Teilnehmerstaaten und nachfolgend vom Programmzentrum des ICP Vegetation daraufhin überprüft, ob sie den Anforderungen nach [24] 
entsprechen [5,6]. In Deutschland erfolgte dies zuvor durch den Arbeitskreis Bioindikation / Wirkungsermittlung der Landesanstalten und -ämter für Umweltschutz: Auffällige Messwerte, die entweder über dem bundesoder dem landesweiten 98. Perzentil lagen, wurden anhand der Metadaten im WebGIS MossMet [25] zusammen mit Geoinformationen u.a. zur Landnutzung in der Umgebung der Moosproben-Entnahmeorte geprüft. Hierzu wurden die über das WebGIS MossMet zugänglichen Probenentnahmeprotokolle und Standortbeschreibungen analysiert sowie Probensammler und ortskundige Fachleute befragt. Ferner wurde ermittelt, ob die Daten derselben Messpunkte bereits bei vorangegangenen Kampagnen auffällig waren. Anschließend wurde eingeschätzt, ob die Werte der regional zu erwartenden Belastung entsprechen. Erschien der gefundene Wert auch danach nicht erklärbar, so wurde dieser nicht weiter berücksichtigt [14].

Expositionsdaten können bewertet werden, wenn man sie z.B. mit Daten über toxikologische Wirkungsschwellen oder mit quasi-natürlichen Hintergrundwerten vergleicht. Zum Abgleich der Daten aus dem MoosMonitoring dienen die Zackenberg Hintergrundwerte [26]. Hierbei handelt es sich um hemisphärische Hintergrundwerte i. S. von [23], die anhand von Moosproben in der Umgebung der Forschungsstation Zackenberg im Nordosten Grönlands gewonnen wurden.

\section{Berechnung der Depositionskarten}

Die Kartierung der Metallkonzentrationen in Moosen erfolgte mit Variogrammanalyse und Kriging-Interpolation. Die Qualität der Flächenschätzungen wurde durch Kreuzvalidierung ermittelt [14]. Die anhand von Emissionsdaten sowie der von EMEP gemessenen und modellierten Gesamtdeposition 2005 [27,28] berechneten Depositionskarten $(50 \mathrm{~km}$ mal $50 \mathrm{~km})$ wurden mit den europaweit geostatistisch geschätzten Flächenkarten zur Cd- bzw. Pb-Konzentration in den 2005 gesammelten Moosen verschnitten. Anschließend wurden die Mediane aller $5 \mathrm{~km}$ mal $5 \mathrm{~km}$ Rasterzellen der $\mathrm{Cd}$ - und $\mathrm{Pb}$ Anreicherungskarten innerhalb der jeweiligen $50 \mathrm{~km}$ mal $50 \mathrm{~km}$ EMEP-Rasterzellen berechnet. Anschließend wurden die Mediane der geschätzten Elementkonzentrationen im Moos sowie die Depositionswerte lntransformiert und korrelations- und regressionsanalytisch untersucht. Die Regressionsfunktionen wurden dann auf die Kriging-Flächenkarten der $\mathrm{Cd}$ - und $\mathrm{Pb}$-Anreicherungen in Moosen angewendet. Die Residuen der beiden Regressionsfunktionen wurden bestimmt, entlogarithmiert, auf die Mittelpunkte der entsprechenden EMEP-Rasterzellen projiziert, variogrammanalytisch auf räumliche Strukturen untersucht und mit Lognormal-Kriging flächenhaft interpoliert. Diese Kriging-Karten der Residuen des $\mathrm{Cd}$ - und
$\mathrm{Pb}$-Regressionsmodells wurden mit den regressionsanalytisch berechneten $\mathrm{Cd}$ - und $\mathrm{Pb}$-Depositionsflächenkarten verrechnet, so dass die durch die beiden linearen Regressionsmodelle bedingten räumlich differenzierten Unter- bzw. Überschätzungstendenzen minimiert wurden. Aus den Europakarten der Cd- und Pb-Gesamtdeposition des Jahres 2005 wurde das Gebiet der Bundesrepublik Deutschland ausgestanzt. Diese Deutschlandkarten zeigen die räumliche Differenzierung der Cdund $\mathrm{Pb}$-Gesamtdeposition als Vielfache der jeweiligen Standardabweichung vom Bundesdurchschnitt. Die dabei gewählte räumliche Auflösung orientiert sich an der Standardabweichung der Distanzen zwischen den Beprobungspunkten in Deutschland und beträgt dementsprechend $5 \mathrm{~km}$ mal $5 \mathrm{~km}$.

Der gewählte Ansatz zur Berechnung von Depositionskarten anhand zweier Informationsquellen, dem EMEPDepositionsmessnetz und dem Moos-Monitoring, hat sich in ähnlich gelagerten Fällen bewährt [29-33]. Die gewählte Verknüpfung erfolgt über das RegressionKriging [34,35] und betrifft zwei aufeinanderfolgende physikalisch-chemische Vorgänge: die atmosphärische Deposition der Schwermetalle $\mathrm{Cd}$ und $\mathrm{Pb}$ sowie deren Anreicherung in Moosen [36]. Regression-Kriging ist eine Technik räumlicher Schätzung, die Regressionsprädiktion mit geostatistischer Interpolation der Regressionsresiduen kombiniert [37].

Die Metallgehalte in den Moosen aus dem Nordosten Grönlands (Zackenberg Hintergrundwerte, [26]) wurden mit den mittleren Metallanreicherungen in den deutschen Bundesländern verglichen. Die Anreicherungsfaktoren wurden bestimmt und am Beispiel des Metalls Pb für die Jahre 1990, 1995, 2000 und 2005 kartiert.

\section{Ergebnisse}

Für die EMEP-Modellierung der Gesamtdeposition von $\mathrm{Cd}$ und $\mathrm{Pb}$ wurde eine Unterschätzung der qualitätskontrollierten EMEP-Messwerten um $20 \%$ bis $30 \%$ berechnet $[28,38]$. Die Qualität der Messungen der Cdund $\mathrm{Pb}$-Konzentrationen in den Moosen wurde anhand von Referenzmaterial kontrolliert. Die gemäß [24] zu erreichenden Werte wurden gut reproduziert [5,6]. Die aus diesen Messwerten des Moos-Monitoring mit Kriging-Interpolation berechneten Europa-Karten weisen klare räumliche Strukturen und befriedigende Schätzqualitäten auf: Für $\mathrm{Cd}$ beträgt der mittlere (Median) korrigierte prozentuale Fehler 22,2 \%, der Pearson Korrelationskoeffizient zwischen Mess- und Schätzwerten beläuft sich auf 0,62, für das Nugget / SillVerhältnis des der Schätzung zugrunde liegenden Variogramm-Modells wurde eine Wert von $63,2 \%$ und für die Aussagereichweite der punktuellen Messungen $64,5 \mathrm{~km}$ berechnet. Für die Flächenschätzung der 




Abbildung 1. Regressionsmodell für die Beziehung zwischen Cd-Gehalten in Moosen (ICP Vegetation Moss Survey 2005) und modellierter Cd-Gesamtdeposition (EMEP-Modellierung für 2005).

Pb-Gehalte in 2005 in Europa gesammelten Moosproben Europas beträgt der Median des korrigierten prozentualen Fehlers 23,7 \%. Der Pearson Korrelationskoeffizient zwischen gemessenen und geschätzten $\mathrm{Pb}$ Werte beträgt 0,63. Für das Nugget / Sill-Verhältnis des der Schätzung zugrundeliegenden Variogramm-Modells wurde der Wert 43,5\% und für die Aussagereichweite $593 \mathrm{~km}$ berechnet [39].

Die statistischen Beziehungen zwischen den $\mathrm{Cd}$ - und $\mathrm{Pb}-$ Gehalten in Moosen und modellierter $\mathrm{Cd}$ Gesamtdeposition (EMEP) wurden nach Verknüpfung der entsprechenden Karten im GIS regressionsanalytisch quantifiziert (Abbildung 1 und Abbildung 2). Die Korrelationskoeffizienten nach Pearson $\left(r_{p}\right)$ und Spearman $\left(r_{S}\right)$ [40] zeigen, dass die Cd-Gehalte in den Moosen etwas schwächer, aber mit $r_{(P, S)} \sim 0,7$ ebenso wie die $\mathrm{Pb}-$ Gehalte in den Moosen mit der Cd- bzw. PbGesamtdeposition $\left(r_{(P, S)} \sim 0,8\right)$ statistisch eng verknüpft sind. Die Regressionsfunktionen wurden auf die Rasterwerte der geostatistisch erzeugten Karten der $\mathrm{Cd}$ - und $\mathrm{Pb}$-Anreicherung in Moosen angewendet, so dass dadurch Karten großräumiger Strukturen der $\mathrm{Cd}$ - und $\mathrm{Pb}$-Gesamtdepositionen im Jahr 2005 erzeugt wurden.

Die Residuen, also der nicht 'erklärte' Anteil der in den beiden linearen Regressionsmodellen quantifizierten Beziehungen zwischen modellierter Gesamtdeposition und Bioakkumulation der Schwermetalle $\mathrm{Cd}$ und $\mathrm{Pb}$ wurden variogrammanalytisch auf räumliche Strukturen untersucht. Die resultierenden Variogramme (Abbildung
3, Abbildung 4) zeigen eine deutliche Autokorrelation der Residuen: Ihr Betrag $(\gamma)$ wächst mit zunehmender Distanz $(h)$ der Moossammelorte voneinander an, und das Nugget / Sill-Verhältnis beträgt 34,4 (Cd) bzw. 33,7 $(\mathrm{Pb})$. Diese von den Regressionsmodellen nicht erfasste und in den regressionsanalytisch erzeugten Karten der $\mathrm{Cd}$ - und Pb-Gesamtdeposition nicht abgebildete Information wird im nächsten Schritt dadurch eingebunden, dass anhand der Residuen-Variogramme Kriging-Karten berechnet wurden, die mit den regressionsanalytisch generierten $\mathrm{Cd}$ - und $\mathrm{Pb}$-Gesamtdepositionskarten durch Addition zu je einer GIS-Karte verrechnet wurden. Aus diesen Karten wurde das Gebiet der Bundesrepublik Deutschland ausgeschnitten, so dass für das Jahr 2005 je eine GIS-Karte der Cd- und Pb-Gesamtdeposition vorliegt (Abbildung 5).

Tabelle 1 enthält statistische Maßzahlen, welche die Ergebnisse der Kartierung der Cd- und Pb-Deposition zusammenfassend beschreiben. Zusammen mit der aus den Karten ersichtlichen räumlichen Differenzierungen der Cd- und Pb-Depositionen ergibt sich folgendes Bild: Räumliche Schwerpunkte oberhalb des bundesweiten Mittelwertes der Cd-Deposition von 0,342 g / ha / a sind im Ruhrgebiet, Südwestdeutschland, Sachsen und Sachsen-Anhalt, Nordwestdeutschland und dem südlichen Schleswig-Holstein zu erkennen. Die räumliche Struktur der Pb-Depositionen, die im Bundesmittel 8,6 g / ha / a betragen, entspricht weitgehend dem der $\mathrm{Cd}$ Depositionen. Allerdings sind für Schleswig-Holstein 


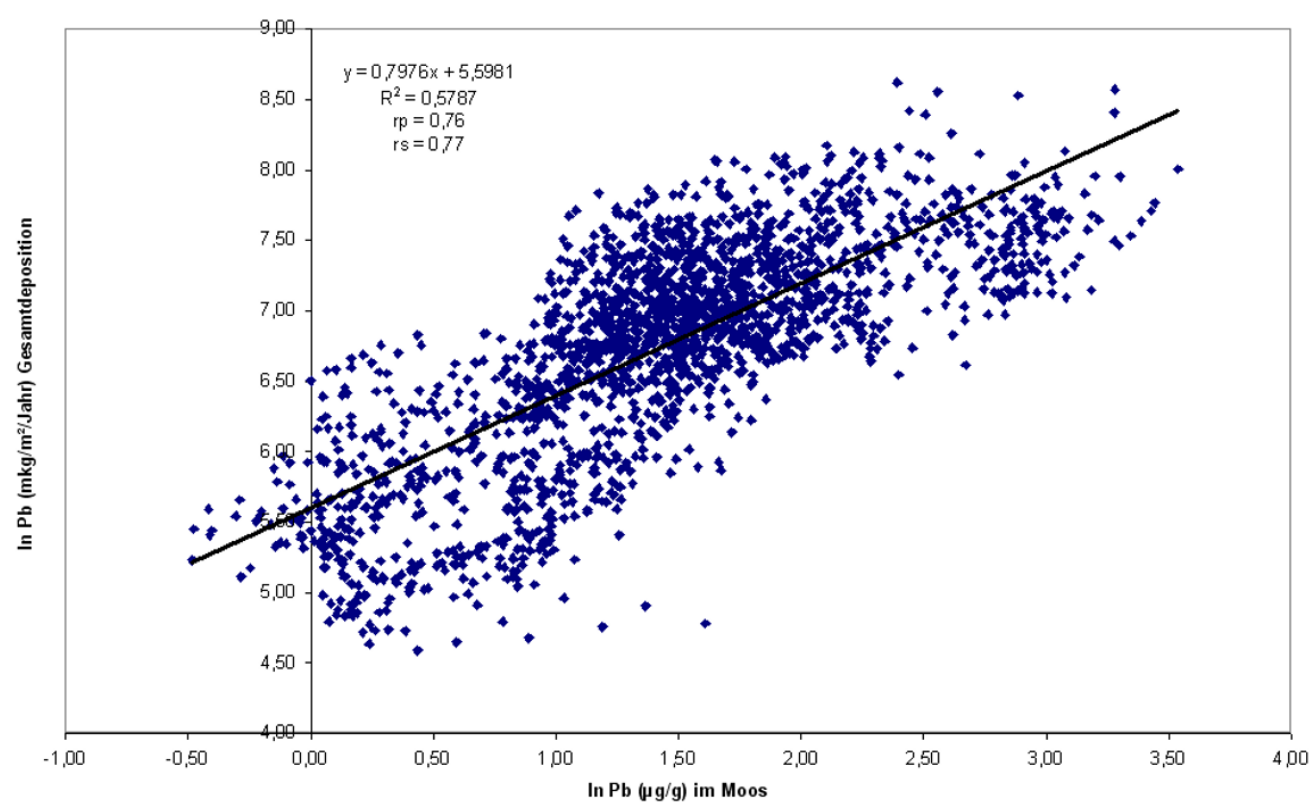

Abbildung 2. Regressionsmodell für die Beziehung zwischen Pb-Gehalten in Moosen (ICP Vegetation Moss Survey 2005) und modellierter Pb-Gesamtdeposition (EMEP-Modellierung für 2005).

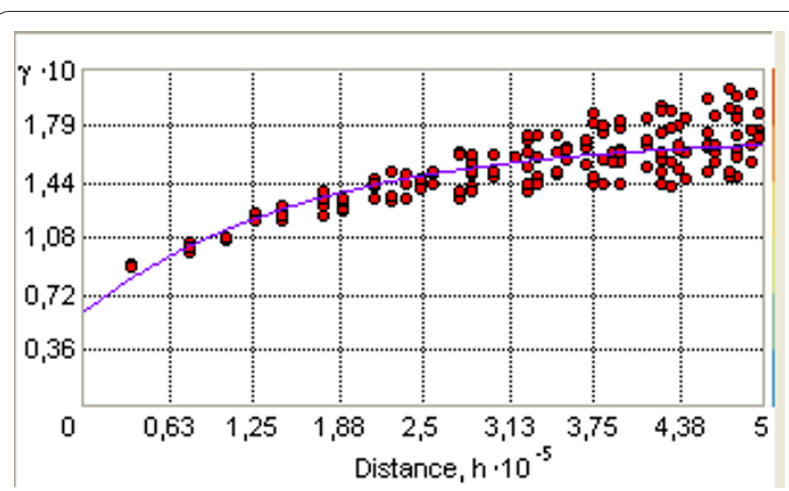

Abbildung 3. Variogramm der Residuen des Regressionsmodells für die Beziehung zwischen Cd-Gehalten in Moosen (ICP Vegetation Moss Survey 2005) und modellierter CdGesamtdeposition (EMEP-Modellierung für 2005).

keine über dem Bundesmittel liegenden Werte berechnet worden.

Tabelle 2 enthält die Anreicherungsfaktoren der Metalle in Moosen, die in den Jahren 1990, 1995, 2000 und 2005 in Deutschland gesammelt wurden, gegenüber denjenigen aus dem Nordosten Grönlands. Demnach bestehen noch heute deutliche Unterschiede in der Anreicherung atmosphärisch deponierter Metalle zwischen Deutschland und Grönland, insbesondere bei $\mathrm{Cd}, \mathrm{Cr}, \mathrm{Cu}, \mathrm{Mo}, \mathrm{Pb}, \mathrm{Sb}, \mathrm{Sn}$, and $\mathrm{Zn}$. Die Abweichungen zwischen den $\mathrm{Pb}$-Anreicherungen in Deutschland und in Grönland wurden - nach Bundesländern differenziert kartiert (Abbildung 6).

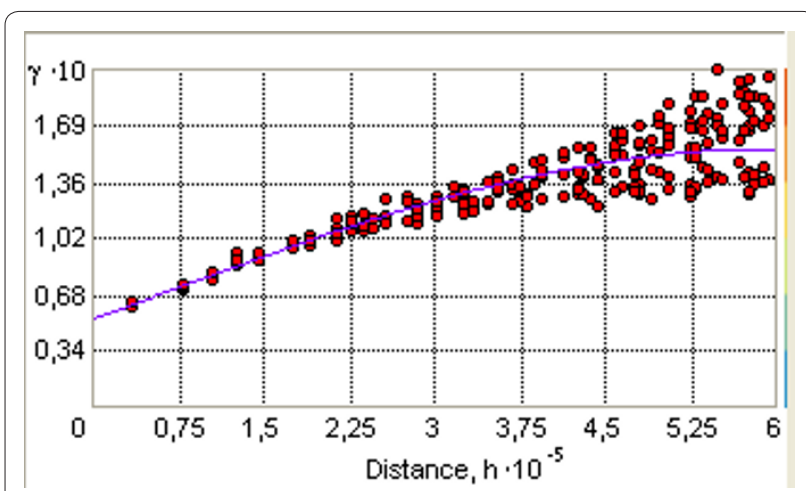

Abbildung 4. Variogramm der Residuen des Regressionsmodells für die Beziehung zwischen Pb-Gehalten in Moosen (ICP Vegetation Moss Survey 2005) und modellierter PbGesamtdeposition (EMEP-Modellierung für 2005).

\section{Diskussion}

Die vor dieser Untersuchung jüngsten Arbeiten zur Berechnung von $\mathrm{Cd}$ - und $\mathrm{Pb}$-Depositionen in Deutschland stammen aus Forschungsvorhaben, die vom Bundesministerium für Umwelt, Naturschutz und Reaktorsicherheit finanziert und vom Umweltbundesamt fachlich begleitet wurden [1,2]. [2] geben lediglich Mittelwerte der $\mathrm{Cd}$ - und $\mathrm{Pb}$-Gesamtdeposition für das Bundesgebiet an. Sie liegen in den Jahren 1995 bis 2004 für Cd zwischen 0,096 und 0,128 g / ha / a. Für denselben Zeitraum wurden für Pb Werte zwischen 3,56 und 4,46 g / ha / a ermittelt. [1] geben den Median der Cd-Gesamtdeposition für ländliche Gebiete mit 0,86 g / ha / a an. 


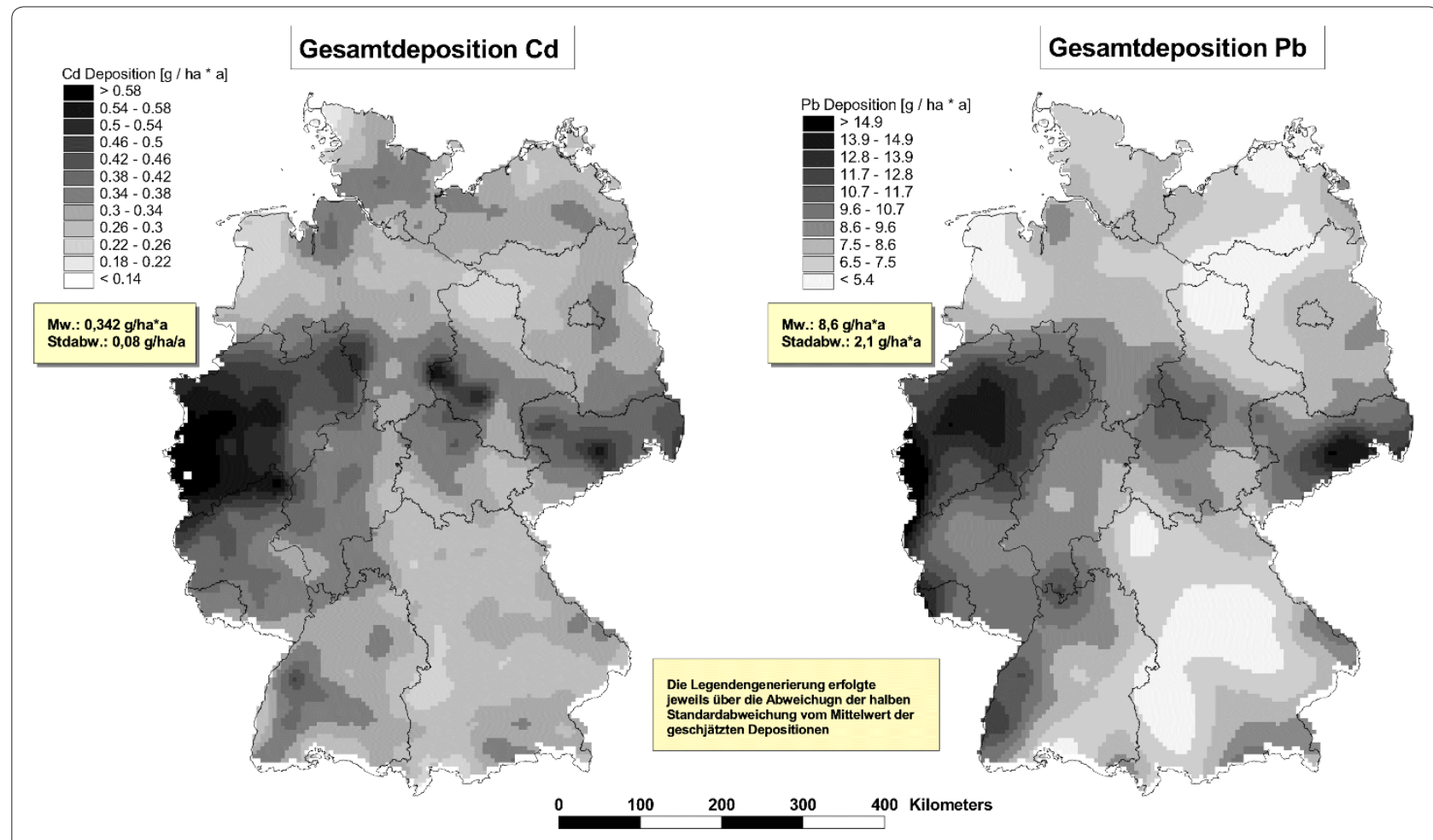

Abbildung 5. Regression-Kriging-Flächenschätzung der Cd- und Pb-Gesamtdeposition in Deutschland (2005) auf Grundlage von Cd-Gehalten in Moosen (ICP Vegetation Moss Survey 2005) und modellierter Cd-Gesamtdeposition (EMEP-Modellierung für 2005), Auflösung $5 \times 5 \mathrm{~km}^{2}$.

Tabelle 1 - Deskriptiv-statistische Maßzahlen der mit Regression-Kriging aus EMEP-Depositionsdaten und Akkumulationsdaten aus dem Europäischen MoosMonitoring berechneten Depositionskarten für $\mathbf{C d}$ und $\mathbf{P b}$

\begin{tabular}{lcc}
\hline Statistische Maßzahl & $\mathbf{C d}$ [g / ha / a] & $\mathbf{P b}$ [g / ha / a] \\
\hline Minimum & 0,15 & 5,6 \\
Maximum & 0,81 & 21,1 \\
Arithmetischer Mittelwert & 0,34 & 8,6 \\
Standardabweichung & 0,08 & 2,1 \\
20. Perzentil & 0,28 & 6,8 \\
50. Perzentil & 0,33 & 8,2 \\
90. Perzentil & 0,45 & 11,5 \\
98. Perzentil & 0,56 & 14,2 \\
\hline
\end{tabular}

Für die Pb-Depositionen in ländlichen Gebieten wird ein Median von 28,7 g / ha / a genannt. Nach den räumlich differenzierten Berechnungen der vorliegenden Untersuchung betrug die Cd-Gesamtdeposition im deutschlandweiten Mittel der Jahre 2003 bis 2005 0,342 g / ha / a bei einer Standardabweichung von 0,08 g / ha / a. Die bundesweit durchschnittliche $\mathrm{Pb}$-Gesamtdeposition betrug 8,6 g / ha / a, die Standardabweichung lag bei 2,1 g / ha / a.
Die Aussagekraft der in dieser Untersuchung berechneten Karten sollte auch durch einen Abgleich mit Messdaten des in Deutschland betriebenen Depositionsmonitoring geprüft werden. Diese Daten waren im Rahmen der beiden letzten Moos-Monitoringprojekte jedoch nicht verfügbar, weder von den Messnetzbetreibern noch vom Umweltbundesamt (UBA). Im Auftrag des UBA wird nicht nur das Moos-Monitoring durchgeführt, sondern auch die Modellierung und Kartierung der Depositionen sowie deren weitere Verwendung für die Berechnung von Critical Loads. Zu diesem Zweck wurde eine Depositionsdatenbank angelegt und in mehreren Forschungsvorhaben fortgeschrieben [2,41-46]. In den genannten Forschungsberichten werden Schwierigkeiten beschrieben, die bei der Zusammenführung der Daten aus mehreren Messnetzen und hinsichtlich ihrer Qualität bestehen. [41] berichtet über die Notwendigkeit, Daten aus Qualitätsgründen auszuschließen, was zur räumlichen Ausdünnung der Daten für geostatistische Modellierungen führt. Weitere Qualitätsprobleme, mit denen sich die Depositionsmodellierung auseinanderzusetzen hat, zeigen [42-45] auf.

Diese Probleme decken sich mit denen, die auf europäischer Ebene bei der Auswertung und 
Tabelle 2. Überschreitungen der Mittelwerte pro Element und Bundesland im Vergleich zu den ZackenbergHintegrundwerten [26] (Ein Faktor von 10 bedeutet, dass der Hintergrundwert um das 10 fache überschritten wurde.)

\begin{tabular}{|c|c|c|c|c|c|c|c|c|c|c|c|c|c|c|c|c|c|c|c|c|}
\hline \multirow[b]{2}{*}{ LAND } & \multicolumn{10}{|c|}{1990} & \multicolumn{10}{|c|}{1995} \\
\hline & Al & As & $\mathrm{Cd}$ & $\mathrm{Cr}$ & $\mathrm{Cu}$ & Mo & $\mathrm{Pb}$ & $\mathrm{Sb}$ & Sn & $\mathrm{Zn}$ & Al & As & $\mathrm{Cd}$ & $\mathrm{Cr}$ & $\mathrm{Cu}$ & Mo & $\mathrm{Pb}$ & $\mathrm{Sb}$ & Sn & $\mathrm{Zn}$ \\
\hline $\mathrm{BB}$ & & & & 5 & 11 & & 124 & & & 10 & 2 & 3 & 7 & 2 & 11 & 6 & 53 & 106 & 21 & 12 \\
\hline $\mathrm{BE}$ & & 3 & 7 & 3 & 9 & & 82 & & & 16 & 1 & 2 & 7 & 2 & 7 & 9 & 51 & 99 & 18 & 14 \\
\hline BW & & 4 & 9 & 3 & 10 & & 102 & & & 10 & 2 & 3 & 9 & 3 & 8 & 8 & 76 & 133 & 37 & 9 \\
\hline BY & & 3 & 6 & 3 & 8 & & 66 & & & 8 & 1 & 2 & 6 & 2 & 10 & 7 & 44 & 95 & 19 & 10 \\
\hline HE & & 3 & 7 & 3 & 7 & & 74 & & & 11 & 2 & 2 & 9 & 3 & 9 & 7 & 47 & 86 & 19 & 11 \\
\hline $\mathrm{HH}$ & & 4 & 7 & 3 & 9 & & 81 & & & 8 & 2 & 4 & 9 & 3 & 13 & 7 & 87 & 170 & 40 & 12 \\
\hline MV & & & & 5 & 10 & & 93 & & & 9 & 2 & 3 & 7 & 2 & 10 & 7 & 42 & 96 & 27 & 12 \\
\hline $\mathrm{NI}$ & & 2 & 6 & 3 & 6 & & 75 & & & 8 & 1 & 2 & 8 & 2 & 7 & 7 & 58 & 95 & 21 & 11 \\
\hline NW & & 4 & 12 & 5 & 11 & & 116 & & & 14 & 1 & 2 & 13 & 5 & 13 & 12 & 83 & 136 & 30 & 17 \\
\hline $\mathrm{RP}$ & & 3 & 7 & 3 & 7 & & 91 & & & 10 & 1 & 3 & 9 & 3 & 9 & 7 & 56 & 99 & 41 & 12 \\
\hline $\mathrm{SH}$ & & 2 & 8 & 2 & 9 & & 74 & & & 10 & 1 & 2 & 7 & 2 & 9 & 6 & 48 & 86 & 19 & 10 \\
\hline$S L$ & & 4 & 9 & 4 & 8 & & 115 & & & 13 & 1 & 3 & 9 & 4 & 8 & 11 & 83 & 87 & 16 & 15 \\
\hline SN & & 12 & 10 & 7 & 12 & & 173 & & & 11 & 2 & 6 & 10 & 2 & 10 & 7 & 90 & 104 & 25 & 14 \\
\hline ST & & 5 & 9 & 5 & 11 & & 111 & & & 10 & 2 & 4 & 7 & 3 & 11 & 8 & 45 & 72 & 24 & 13 \\
\hline \multirow[t]{2}{*}{$\mathrm{TH}$} & & 5 & 7 & 4 & 8 & & 98 & & & 7 & 2 & 3 & 8 & 3 & 9 & 7 & 67 & 104 & 19 & 10 \\
\hline & \multicolumn{10}{|c|}{2000} & \multicolumn{10}{|c|}{2005} \\
\hline LAND & Al & As & $\mathrm{Cd}$ & $\mathrm{Cr}$ & $\mathrm{Cu}$ & Mo & $\mathrm{Pb}$ & $\mathrm{Sb}$ & Sn & $\mathrm{Zn}$ & Al & As & $\mathrm{Cd}$ & $\mathrm{Cr}$ & $\mathrm{Cu}$ & Mo & $\mathrm{Pb}$ & $\mathrm{Sb}$ & Sn & $\mathrm{Zn}$ \\
\hline BB & 1 & 2 & 5 & 1 & 7 & & 28 & 76 & & 9 & 1 & 2 & 5 & 5 & 7 & 10 & 21 & 74 & & 9 \\
\hline $\mathrm{BE}$ & 1 & 1 & 4 & 1 & 5 & & 20 & 60 & & 9 & & & & & & & & & & \\
\hline BW & 2 & 2 & 7 & 2 & 7 & & 45 & 95 & & 8 & 1 & 2 & 5 & 3 & 7 & 11 & 30 & 96 & & 8 \\
\hline BY & 1 & 1 & 4 & 1 & 7 & & 23 & 79 & & 7 & 1 & 1 & 5 & 3 & 7 & 9 & 20 & 84 & & 8 \\
\hline $\mathrm{HE}$ & 1 & 1 & 6 & 1 & 7 & & 27 & 84 & & 8 & 1 & 2 & 6 & 6 & 8 & 17 & 30 & 108 & & 11 \\
\hline $\mathrm{HH}$ & 1 & 2 & 5 & 2 & 10 & & 36 & 154 & & 9 & 1 & 1 & 3 & 9 & 12 & 15 & 19 & 83 & & 10 \\
\hline MV & 1 & 2 & 6 & 1 & 8 & & 29 & 67 & & 10 & 1 & 2 & 5 & 14 & 9 & 22 & 19 & 62 & & 12 \\
\hline $\mathrm{Nl}$ & 1 & 1 & 6 & 1 & 6 & & 27 & 82 & & 8 & 1 & 1 & 5 & 5 & 6 & 12 & 23 & 81 & & 9 \\
\hline NW & 1 & 1 & 9 & 2 & 9 & & 49 & 124 & & 13 & 1 & 2 & 9 & 4 & 10 & 16 & 45 & 131 & & 16 \\
\hline $\mathrm{RP}$ & 2 & 2 & 7 & 2 & 7 & & 49 & 94 & & 11 & 1 & 2 & 7 & 3 & 8 & 11 & 32 & 94 & & 11 \\
\hline SH & 1 & 2 & 6 & 1 & 7 & & 34 & 78 & & 8 & 1 & 1 & 5 & 5 & 7 & 12 & 22 & 67 & & 14 \\
\hline $\mathrm{SL}$ & 1 & 3 & 6 & 2 & 8 & & 56 & 77 & & 12 & 1 & 2 & 6 & 8 & 7 & 22 & 33 & 44 & & 14 \\
\hline SN & 1 & 3 & 7 & 1 & 7 & & 42 & 88 & & 9 & 2 & 5 & 7 & 8 & 9 & 18 & 39 & 103 & & 12 \\
\hline ST & 2 & 2 & 6 & 2 & 8 & & 33 & 65 & & 10 & 1 & 2 & 7 & 6 & 8 & 18 & 28 & 61 & & 13 \\
\hline $\mathrm{TH}$ & 2 & 2 & 6 & 2 & 8 & & 42 & 88 & & 8 & 2 & 2 & 5 & 4 & 7 & 10 & 30 & 85 & & 8 \\
\hline
\end{tabular}

Modellierung von Depositionen auftreten: Von 860 ICP Forests Level II-Flächen standen [12] für Auswertungen Depositionsdaten (bulk, throughfall) von 185 bzw. 249 Standorten mit maximal 30 fehlenden Messungen pro Jahr im Zeitraum 2000 bis 2005 zur Verfügung. [47] mussten Daten von 160 ICP-Forests MonitoringStandorten in sieben europäischen Ländern von einer Untersuchung ausschließen, in der die im EMEP modellierten Depositionen anhand von Messwerten überprüft werden sollten. Qualitätssicherung, die Harmonisierung der Messprogramme hinsichtlich räumlicher und zeitlicher Kriterien sowie die Methodenstandardisierung sind weiterhin Herausforderungen des Forstmonitoring [48].

In der hier vorgestellten Studie wurde vereinbart, die im europäischen Moosmonitoring des ICP Vegetation an bis zu 7000 Orten in Europa erhobenen Daten über die Schwermetallgehalte in Moosen mit den im EMEP modellierten $\mathrm{Cd}$ - und $\mathrm{Pb}-$ Gesamtdepositionen $\mathrm{zu}$ verknüpfen und die statistischen Beziehungen zwischen den 


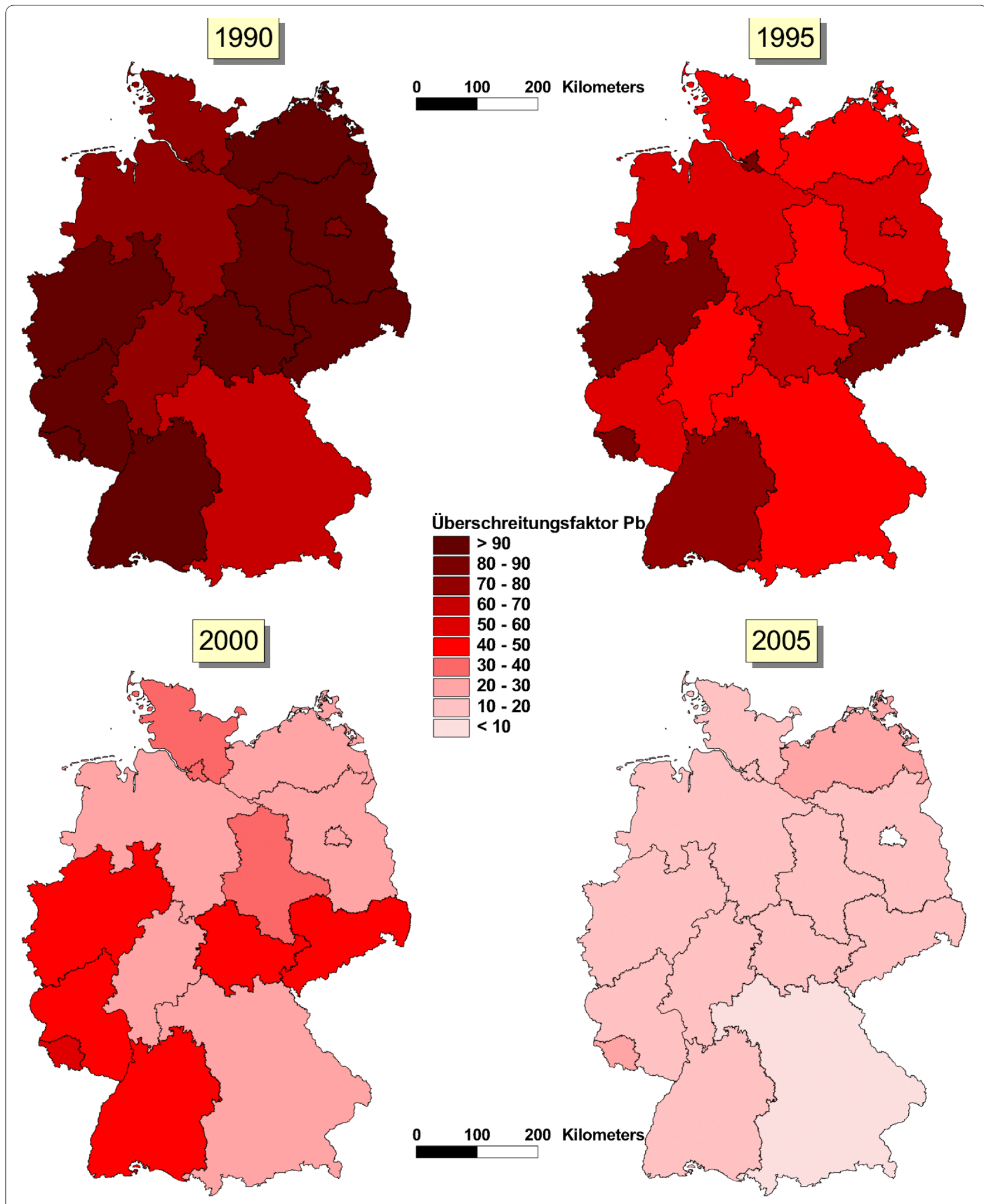

Abbildung 6. Räumliche und Zeitliche Differenzierung der Abweichungen der Bioakkumulationen für Pb in Moosen in Deutschland von den Zackenberg-Hintergrundwerten [26]. 
Datensätzen daraufhin zu überprüfen, ob die Moosdaten eine statistische Hilfsvariable für die Berechnung von räumlich hoch aufgelösten Depositionskarten sein können, so wie dies in ähnlichen Zusammenhängen auch erfolgt [29-33]. Der Vorteil des Moos-Monitoring besteht darin, dass das experimentelle Design etwa im Vergleich zum Forst-Monitoring sehr viel weniger komplex angelegt ist und die Qualitätskontrolle vom Programmstart an von der Probenentnahme bis zur Datenauswertung reichte, also integraler Bestandteil des Monitoring-Konzeptes war. Damit ist eine zentrale Forderung von [15] an Umweltmonitoring-Programme erfüllt. ICP Forests bereitet die Qualitätssicherung insbesondere bei den Probeneinsammlungen und bei der Datenzusammenführung und Auswertung noch immer Probleme [16].

[1] weisen darauf hin, dass mit dem Moos-Monitoring ein höherer Anteil der Gesamtdeposition als über wetonly- und Bulk-Sammler gemessen werden kann. Im Gegensatz zu den Methoden auf Grundlage technischer Sammler werden mit Moos-Monitoring auch rezeptorabhängige Einträge erfasst. Es können damit sowohl die feuchte Deposition (Nebel. Tau, Reif) als auch Anteile der trockenen Deposition (Sedimentation und Trägheitsabscheidung durch Filterwirkung, diffusiver Eintrag), die sich weder mit wet only-Sammlern noch mit bulkSammlern messen lassen, abgebildet werden. Die ermittelten Werte gelten für Rezeptoren mit ähnlicher Struktur und Oberfläche. Werden die Moosproben räumlich benachbart zu bulk- oder wet only-Sammlern betrieben, könnten gegebenenfalls über die Korrelationen flächendeckende Abschätzungen der einzelnen Beiträge gemacht werden. Jedoch habe sich das Moos-Monitoring zur Berechnung der Stoffeinträge in Böden als nicht brauchbar erwiesen, da die Korrelationsanalysen zwischen Mooskonzentrationen und wet-only und auch Bodenhorizonten bis auf wenige Standorte keinen klaren Zusammenhang zeigten. Die Beeinflussung der Moose sei „offensichtlich sehr von lokalen Gegebenheiten abhängig, ohne sie benennen zu können bzw. über die Jahre Gesetzmäßigkeiten feststellen zu können“ [1]. Hierzu ist Folgendes anzumerken:

1. Die Korrelationsberechnungen wären nach den Aussagen von [1] vorzugsweise mit bulk-Depositionswerten durchzuführen gewesen. Solche Berechnungen sind im Moos-Monitoring erfolgt. Es zeigte sich, dass die Rangkorrelationskoeffizienten $\left(r_{s}\right)$ zwischen den Metallkonzentrationen in Moosen einerseits und der Deposition dieser Elemente innerhalb und außerhalb von Wäldern sowie modellierter Nass-, Trocken- und Gesamtdeposition andererseits nach Stoffen und Depositionsmechanismus variieren: Hohe und sehr hohe Korrelationen bestehen zwischen den Konzentrationen von As und $\mathrm{Cr}$ in Moosen und in der wet only-Deposition im Freiland (As $r_{s} 0.6$ und 0.7, Cd 0.9 und 1.0, EMEP/ UBA-Messnetz, Messnetz ICP Integrated Monitoring ) $[14,49]$. Mittlere Korrelationen weisen die Konzentrationen von $\mathrm{Cd}, \mathrm{Cu}, \mathrm{Pb}$, and $\mathrm{Zn}$ in Moosen und bulkDepositionen auf (ICP Forests Level II). Die statistischen Beziehungen zwischen den Konzentrationen in bulkBestandesdepositionen (ICP Forests Level II) und Moosen sind bei $\mathrm{Cd}, \mathrm{Cu}$ and $\mathrm{Pb}$ gering, mittelstark hingegen für $\mathrm{Zn}$. Die Korrelationen von $\mathrm{Cd}$ and $\mathrm{Pb}$ in Moosen und den modellierten Depositionen [44] sind im Gegensatz zu den dieser Untersuchung zugrundeliegenden (Abbildung 1 und 2) niedrig bis mittel und variieren zeitlich, stoffspezifisch und nach modelliertem Depositionsprozess: Die höchsten Korrelationen bestehen zwischen den Konzentrationen von $\mathrm{Cd}$ und $\mathrm{Pb}$ in Moosen und in der modellierten Gesamt- und Trockendeposition [14,49].

2. Bodendaten wurden dem Moos-Monitoring für Korrelationsanalysen nicht zur Verfügung gestellt, so dass die Aussagen von [1] (2008, S. C 84) nicht überprüft werden konnten. Wichtig bei diesen Korrelationsberechnungen ist es, dass man räumlich benachbart erhobene Daten miteinander korreliert. Ob dies beachtet wurde, ist den Ausführungen von [1] nicht zu entnehmen.

3. Nicht nur die atmosphärische Abscheidung von Stoffen auf Moosen ist von lokalen Gegebenheiten abhängig. Dies gilt gleichermaßen für alle Sammleroberflächen, seien es technische oder natürliche. Seit Mitte der 1980er Jahre ist bekannt, dass selbst in Sammlern eines Typs an einem Standort z.T. sehr unterschiedliche Messwerte erzielt werden [50-52]. [53] zeigen, dass Bergerhoff-Depositionssammler nur für die Depositionssammlung in urban-industriellen Gebieten mit hohen Raten überwiegend trockener Deposition geeignet sind und keinesfalls für die Erfassung des Stoffeintrags in Hintergrundgebieten. Insofern ist es fachlich bedenklich, die Messdaten, die auf unterschiedlichen Sammlertypen basieren, bei der Depositionsmodellierung miteinander $\mathrm{zu}$ verrechnen oder den Schwermetallindikator der Länderinitiative Kernindikatoren (LIKI) aus den bislang nicht zusammengeführten Daten des 46 Hintergrund-Messstellen umfassenden Bergerhoff-Sammlernetzes ableiten zu wollen. Dies gilt umso mehr, als dieses Messnetz im Gegensatz zum Moos-Monitoring weder die Flächenproportionen der Bundesländer berücksichtigt noch naturräumlich repräsentativ ist.

4. Anders als für die Depositionsmessungen, auf denen die Berechnungen von [2] sowie [1] beruhen, gibt es sehr wohl systematische Untersuchungen darüber, auf welcher räumlichen Maßstabsebene welche Faktoren mit den in den Moosen angereicherten Stoffe korrelieren. Dies ist dadurch möglich, dass die Merkmale der Moosprobenentnahmeorte und ihrer weiteren Umgebung genauso 
wie die Messdaten im WebGIS MossMet [25] zusammengeführt und auf Zusammenhänge hin untersucht werden. Die dabei festgestellten statistischen „Gesetzmäßigkeiten" [1] sind veröffentlicht [49,54-57].

Inwieweit das Moos-Monitoring oder andere Messnetze wie z.B. die Depositionsmessnetze tatsächlich Hintergrundwerte erfassen, hängt zum einen von der Definition und zum anderen von der Operationalisierung des Begriffes „Hinter-grundwert“ ab. Eine allgemein akzeptierte darüber hinausgehende operationale Definition des Begriffes in Bezug auf atmosphärische Depositionen ist nicht etabliert [58]. Die in Europa gemessene Metall-Bioakkumulation können mit quasinatürlichen Hintergrundwerten der Nordhemisphäre, den in Nordost-Grönland gewonnenen ZackenbergHintergrundwerten [26], verglichen und bewertet werden. Diese Referenzwerte beziehen sich auf die Umgebung der Forschungsstation Zackenberg, wo an acht Standorten Proben der Moosarten Sphagnum olafii (Flatberg) and Aulacomnium turgidum (Wahlenberg) Schwägr. gesammelt und auf elf Metalle (inkl. Cd und $\mathrm{Pb}$ ) chemisch analysiert (Tabelle 2) wurden. Für die $\mathrm{Cd}$ - und $\mathrm{Pb}-$ Konzentration in den Moosen konnte sich so eine mittlere Elementkonzentration von $0,041 \mu \mathrm{g} / \mathrm{g}$ für $\mathrm{Cd}$ und $0,16 \mu \mathrm{g} / \mathrm{g}$ für $\mathrm{Pb}$ berechnen lassen. Bei diesem Vergleich muss allerdings berücksichtigt werden, dass die von [26] beprobten Moosarten in den europaweiten Moos-Monitoring-Kampagnen nicht $\mathrm{zu}$ den prioritären Moosarten zählen. [59] gibt für die im Nordosten Grönlands gesammelten Bult-Sphagnen eine leicht erhöhte Aufnahmekapazität gegenüber den prioriären Moosarten des europäischen Moos-Monitorings an, weshalb die Hintergrundwerte für diese Arten gegenüber den Zackenberg-Hintergrundwerten eher etwas niedriger anzusetzen sind.

Der mit dem Critical Loads-Ansatz verfolgte Weg, PEC / PNEC-Verhältnisse für atmosphärische Stoffeinträge auf Ökosystemebene räumlich differenzierend zu berechnen, ist sinnvoll. Dabei spielt die Modellierung der PEC-Werte in Form atmosphärischer Depositionen eine wesentliche Rolle. Die Inputdaten für die Modellierung sind dabei Depositionsmessungen aus unterschiedlichen Messprogrammen in Deutschland, deren Designs in räumlicher und zeitlicher Hinsicht sowie in Bezug auf die gemessenen Komponenten und Messmethoden nicht hinreichend harmonisiert sind. Hinzu kommen Schwierigkeiten beim Zusammenführen der Daten für die Depositionsmodellierung sowie Probleme mit der räumlichen Dichte der Depositionsmessnetze. Zusammen mit den prinzipiell Mess- und Modellierungsverfahren inhärenten Unschärfen führt dies dazu, dass sich die modellierten Werte von Messdaten zum Teil erheblich unterscheiden. Ob sich diese Schwierigkeiten mindern lassen, wenn man die Schwermetallanreicherung als physikalisch und chemisch mit ihrer atmosphärischen Deposition gekoppelte Hilfsvariable bei der Berechnung von Depositionskarten verwendet, wurde in dieser Arbeit mit positivem Ergebnis geprüft. Hierzu standen qualitätskontrollierte Messdaten aus dem EMEPDepositionsmessnetz sowie Daten aus dem Europäischen Moos-Monitoring des UNECE ICP Vegetation zur Verfügung.

Die Modellierung atmosphärischer Depositionen von $\mathrm{Cd}$ und $\mathrm{Pb}$ in Deutschland sollte dem Vorbild der Kooperation zwischen EMEP-Depositionsmodellierung und ICP Vegetation Moos-Monitoring folgen und prüfen, ob sich die Ergebnisse der Depositionsmodellierung in Deutschland durch Koppelung mit den Daten aus dem Moos-Monitoring validieren lassen. Hierzu wäre es erforderlich, die Depositionsdatenbank [2,41-46] mit dem WebGIS MossMet [25] zu verknüpfen. Dabei wären auch die Informationen, mit denen die Standorte der Depositionsmessungen und die Messungen selbst beschrieben werden, einzupflegen. Solche Metadaten sind für die Moos-Monitoringkampagnen dokumentiert und werden in die statistischen Analysen einbezogen.

\section{Competing interests}

No competing interests do exist.

\section{Authors' contributions}

WS wrote the text. MH and RP conducted the computations. $\mathrm{HZ}, \mathrm{HF}$ and II supported the work by dealing with the validity of experimental and modelling data.

\section{Danksagung}

Wir danken dem United Kingdom Department for Environment, Food and Rural Affairs (Defra; contract AQ0810. LEP 0901), der UNECE (Trust Fund) und dem Natural Environment Research Council (NERC) für die Finanzierung des Koordinationszentrums des ICP Vegetation Programms am Centre for Ecology and Hydrology (CEH) Bangor, UK. Ferner danken wir den Institutionen für die Bereitstellung der Ergebnisse der nationaler Moos-Monitoring-Kampagnen aus Italien (Renate Alber, Environmental Agency of Bolzano, Laives, Italien), der Türkei (Mahmut Coşkun, Canakkale Onsekiz Mart University, Faculty of Medicine Department of Medical Biology, Çanakkale), Belgien (Ludwig De Temmerman, Veterinary and Agrochemical Research Centre, Tervuren), Lettland (Marina Frolova, Latvian Environment, Geology and Meteorology Agency, Riga), Spanien (Laura González-Miqueo and Jesús M. Santamaría, Departmento de Quimica y Edafologia, Universidad de Navarra, Navarra), Slowenien (Zvonka Jeran, Department of Environmental Sciences, Institut Jozef Stefan; Primož Simonèiè Slovenian Forestry Institute), Finnland (Eero Kubin and Juha Piispanen, Finnish Forest Research Institute, Muhos Research Station, Muhos), Frankreich (Sébastien Leblond, Muséum National d'Histoire Naturelle, Paris), Estland (Siiri Liiv, Tallinn Botanic Garden, Tallinn), der Slowakei (Blanka Maňkovská, Institute of Landscape Ecology, Slovak Academy of Science, Bratislava), Schweden (Åke Rühling), Russland (Marina Frontasyeva, Frank Laboratory of Neutron Physics, Joint Institute for Nuclear Research, Dubna), Norway (Eiliv Steinnes, Department of Chemistry Norwegian University of Science and Technology, Trondheim), Weißrussland (Yuliya Aleksiayenak, International Sakharov Environmental University, Minsk), Ukraine (Oleg Blum, National Botanical Garden Academy of Science of Ukraine, Kyiv), Denmark (Maria Dam, Food, Veterinary and Environmental Agency, Tórshavn), Serbien (Miodrag Krmar, Physics Department, Faculty of Sciences, University Novi Sad, Novi Sad), Litauen (Kestutis Kvietkus, Institute of Physics, Vilnius), Island (Sigurður Magnússon, Icelandic Institute of Natural History, Reykjavík), Mazedonien (Viktor Urumov, Saints Cyril and Methodius University, Faculty of Natural Sciences and Mathematics Institute of Physics, Skopje), Polen (Krystyna Grodzińska, Institute of Botany, Polish Academy of Sciences, Krakow; Szymon 
Korzekwa, University of Opole, Opole), Tschechien (Ivan Suchara, Silva Tarouca Research Institute for Landscape and Ornamental Gardening), Bulgarien (Lilyana Yurukova, Institute of Botany, Bulgarian Academy of Sciences, Sofia), der Schweiz (Lotti Thöni, FUB - Research Group for Environmental Monitoring, Rapperswil) und Österreich (Harald G. Zechmeister, Faculty of Life Sciences, University of Vienna, Vienna).

\section{Author details}

'Chair of Landscape Ecology, University of Vechta, P.O.B. 1553, 49364 Vechta, Germany. ${ }^{2}$ Universität Wien, Rennweg 14, 1030 Wien, Österreich. ${ }^{3}$ Centre for Ecology \& Hydrology, Environment Centre Wales, Deiniol Road, Bangor, Gwynedd, LL57 2UW, UK. "Meteorological Synthesizing Centre-East of EMEP, Krasina pereulok, 16/1, 123056 Moscow, Russia.

Received: 12 April 2011 Accepted: 18 May 2011

Published: 18 May 2011

\section{Zitierte Literatur}

1. Knappe F, Möhler S, Ostermayer A, Lazar S, Kaufmann C: Vergleichende Auswertung von Stoffeinträgen in Böden über verschiedene Eintragspfade. UBATexte 36/08. Dessau, 2008.

2. Gauger T, Haenel H-D, Rösemann C, Dämmgen U, Bleeker A, Erisman JW, Vermeulen AT, Schaap M, Timmermanns RMA, Builtjes PJH, Duyzer JH: National Implementation of the UNECE Convention on Long-range Transboundary Air Pollution (Effects) Part 1: Deposition Loads: Methods, modelling and mapping results, trends. UBA-Texte 38/08. Dessau; 2008.

3. Escher $\mathrm{Bl}$, Hermens JL: Internal exposure, linking bioavailability to effects. Environ Sci Technol2004, 38:455A-462A.

4. Worms I, Simon DF, Hassler CS, Wilkinson KJ: Bioavailability of trace metals to aquatic microorganisms, importance of chemical, biological and physical processes on biouptake. Biochimie 2006, 88:1721-173.

5. Harmens H, Norris D and the participants of the moss survey: Spatial and temporal trends in heavy metal accumulation in mosses in Europe (1990-2005). Programme Coordination Centre for the ICP Vegetation, Centre for Ecology and Hydrology, Bangor, UK; 2008. [http://icpvegetation.ceh.ac.uk]. Accessed 18 November 2009.

6. Harmens H, Norris DA, Steinnes E, Kubin E, Piispanen J, Alber R, Aleksiayenak Y, Blum O, Coşkun M, Dam M, De Temmerman L. Fernández, JA, Frolova M Frontasyeva M, González-Miqueo L, Grodzińska K, Jeran Z, Korzekwa S, Krmar M, Kvietkus K, Leblond S, Liiv S, Magnússon SH, Maňkovská B, Pesch R, Rühling Å, Santamaria JM, Schröder W, Spiric Z, Suchara I, Thöni L, Urumov V, Yurukova L, Zechmeister HG: Mosses as biomonitors of atmospheric heavy metal deposition: spatial and temporal trends in Europe. Environ Pollut 2010, 158: 3144-3156.

7. Selck H, Forbes VE: The relative importance of water and diet for uptake and subcellular distribution of cadmium in the depositfeeding polychaete. Capitella sp. I. Mar Environ Res 2004, 57:261-279.

8. AhlfW, Drost W, Heise S: Incorporation of metal bioavailability into regulatory frameworks - metal exposure in Water and sediment. J Soils Sediments 2009, 9:411-419.

9. Worms I, Simon DF, Hassler CS, Wilkinson KJ: Bioavailability of trace metals to aquatic microorganisms, importance of chemical, biological and physical processes on biouptake. Biochimie 2006, 88:1721-1731.

10. Förstner U: Sediments and priority substances in river basins - new directive 2008/105/EC; sediment issues in management plans. J Soils Sediments 2009, 9:89-93.

11. Doyle U, Heiß C: Bewertung von diffusen Stoffeinträgen im Rahmen der nationalen Biodiversitätsstrategie. Renaissance der integrierten Umweltbeobachtung. Umweltwiss Schadst Forsch 2009, 21:539-548.

12. Lorenz M, Granke O: Deposition measurements and critical loads calculations: monitoring data, results and perspective. iForest 2009, 2:11-14.

13. Spranger T, Kunze F, Gauger T, Nagel D, Bleeker A, Draaijers G: Critical loads exceedances in Germany and their dependence on the scale of input data. Water Air Soil Pollut 2001, Focus 1:335-351.

14. Schröder W, Pesch R, Matter Y, Göritz A, Dieffenbach-Fries H, Genßler L: Trend der Schwermetall-Bioakkumulation 1990 bis 2005: Qualitätssicherung bei Probenahme, Analytik, geostatistischer Auswertung. Umweltwiss Schadst Forsch 2009, 21:549-574.

15. Ferretti M: Forest health assessment and monitoring - Issues for consideration. Environ Monit Assess 1997, 48:45-72.

16. Ferretti M, König N, Rautio P, Sase H: Quality assurance (QA) in international forest monitoring programmes: activity, problems and perspectives from East Asia and Europe. Ann For Sci 2009, 66:403/1.-403/12.

17. Wuyts K, De Schrijver A, Verheyen K: The importance of forest type when incorporating forest edge deposition in the evaluation of critical load exceedance. iForest 2009, 2: 43-45.

18. Bealey W J, Long S, Spurgeon D J, Leith I, Cape I N: Review and implementation study of biomonitoring for assessment of air quality outcomes. Science Report - SC030175/SR2. Environment Agency; Bristol 2008:1-170.

19. ICP Vegetation: Heavy metals in European mosses: 2005/2006 survey. Monitoring manual. ICP Vegetation Coordination Centre, Centre for Ecology and Hydrology, Bangor, UK; 2005. [http://icpvegetation.ceh.ac.uk]. Accessed 26 January 2010.

20. Pesch R, Schröder W, Dieffenbach-Fries H, Genßler L, Kleppin L: Optimierung des Moosmonitoring-Messnetzes in Deutschland. Umweltwiss Schadst Forsch 2008, 20:49-61.

21. O'Connor P: Monitoring - Quality assurance and quality control. Government of South Australia. South Australian Murray-Darlin Basin. Natural Resources Management Board; 2009. [http://www.samdbnrm.sa.gov. au/Portals/7/Monitoring\%20Quality\%20Assurance\%20and\%20Quality\%20 Control\%20(C).pdf]. Accessed 25 November 2009.

22. Mohr K, Holy M, Pesch R, Schröder W: Bioakkumulation von Metallen und Stickstoff zwischen 1990 und 2005 in Niedersachsen. Umweltwiss Schadst Forsch 2009, 21:459-464.

23. Szepesi DJ, Fekete KE: Background levels of air and precipitation quality for Europe. Atmosph Environ, 21: 1623-1630.

24. Steinnes E, Rühling Å, Lippo H, Mäkinen A: Reference materials for largescale metal deposition surveys. Accredit Qual Assur, 2:243-249.

25. Kleppin L, Schröder W, Pesch R, Schmidt G: Entwicklung und Erprobung einer Metadaten- und WebGIS-Applikation für das Expositionsmonitoring mit Moosen in Deutschland. Ein Beitrag zum LTER-Netzwerk. Umweltwiss Schadst Forsch 2008, 20:38-48.

26. Zechmeister Hg, Dullinger S, Köllensperger G, Ertl S, Lettner C, Reiter K: Do metal concentrations in moss from the Zackenberg area, Northeast Greenland provide a baseline for monitoring? Environ Sci Pollut Res 2010, 13, 398-405.

27. Aas W, Breivik K: Heavy metals and POP measurements, 2006. EMEP/CCC-Report $4 / 2008$.

28. Ilyin I, Rozovskaya O, Travnikov O, Aas W, Hettelingh JP, Reinds GJ: Heavy Metals: Transboundary Pollution of the Environment. EMEP Status Report 2/2008.

29. Bertino L,Wackernagel H: Case studies of change-of-support problems. Technical report N-21/02/G, ENSMP_ARMINES. Centre de Géostatistique, Fontainebleau, France; 2002

30. Genikhovich E, Filatova E, Ziv A: A method for mapping the air pollution in cities with the combined use of measured and calculated concentrations. Int J Environ Pollut 2002, 18:56-63.

31. Goovaerts P: Geostatistical approaches for incorporating elevation into the spatial interpolation of rainfall. J Hydrol 2000, 228:113-129.

32. Pauly M, Drueke M: Mesoscale spatial modelling of ozone immissions. An application of geostatistical methods using a digital elevation model. Gefahrstoffe- Reinhalt Luft 1996, 56:225-230

33. Van de Kassteele J, Stein A, Dekkers ALM, Velders GJM: External drift kriging of NOx concentrations with dispersion model output in a reduced air quality monitoring network. Environ Ecol Stat 2009, 16:321-339.

34. Hengl T, Heuvelink GBM, Rossiter DG: About regression-kriging: From equations to case studies. Comput Geosci 2007, 33:1301-1315.

35. Odeh I OA, McBratney AB, Chittleborough DJ: Further results on prediction of soil properties from terrain attributes: heterotopic cokriging and regression-kriging. Geoderma 1995, 67:215-226.

36. Berg T, Steinnes E: Use of mosses (Hylocomium splendens and Pleurozium schreberi) as biomonitors of heavy metal deposition. From relative to absolute deposition values. Environmental Pollution 1997, 98: 61-71.

37. Vanwalleghem T, Meentemeyer RK: Predicting forest microclimate in heterogeneous landscapes. Ecosystems 2009, 12:1158-1172

38. Ilyin I, Travnikov O: Modelling of heavy metal airborne pollution in Europe: evaluation of the model performance. EMEP/MSC-E Technical Report 8/2005. Meteorological Synthesizing Centre - East, Moscow, Russian Federation; 2005 [http://www.msceast.org]. Accessed 19. November 2009].

39. Schröder W, Holy M, Pesch R, Harmens H, Ilyin I, Alber R, Aleksiayenak Y, Blum O, Coşkun M, Dam M, De Temmerman L, Frolova M, Frontasyeva M, Gonzalez Miqueo L, Grodzińska K, Jeran Z, Korzekwa S, Krmar M, Kubin E, Kvietkus K, 
Leblond S, Liiv S, Magnússon S, Maňkovská B, Piispanen J, Rühling Å, Santamaria J, Simoncic P, Steinnes E, Suchara I, Thöni L, Urumov V, Yurukova L, Zechmeister HG: Are cadmium, lead and mercury concentrations in mosses across Europe primarily determined by atmospheric deposition of these metals. J Soils Sediments 2010, 10:1572-1584.

40. Myers JL, Arnold DW: Research Design and Statistical Analysis. $2^{\text {nd }}$ ed. Lawrence Erlbaum. Mahwah, New Jersey, USA: 2003:1-508.

41. Gauger T: Nationale Luftreinhaltestrategie - Umsetzung von EU-Anforderungen; Teilvorhaben 02: Aufbereitung, Nutzung und Weiterentwicklung nationaler, hochauflösender Datensätze zu Konzentrationen und Depositionen von Luftschadstoffen. Forschungsvorhaben im Auftrag des BMU/UBA, FE-Nr. 203 43 257/02. 23 S. Text + 54 S. Anhang. FAL-AOE, Braunschweig; 2005.

42. Gauger T, Anshelm F, Köble R: Kritische Luftschadstoff-Konzentrationen und Eintragsraten sowie ihre Überschreitung für Wald und Agrarökosysteme sowie naturnahe waldfreie Ökosysteme. Teil 1: Deposition Loads. Forschungsvorhaben im Auftrag des BMU / UBA, FE-Nr. 29785 079. Institut für Navigation, Universität Stuttgart. Berlin, Stuttgart; 2000

43. Gauger T, Anshelm F, Köble R: Kritische Luftschadstoff-Konzentrationen und Eintragsraten sowie ihre Überschreitung für Wald und Agrarökosysteme sowie naturnahe waldfreie Ökosysteme. Teil 2: Critical Levels. Forschungsvorhaben im Auftrag des BMU / UBA, FE-Nr. 29785 079. Institut für Navigation, Universität Stuttgart. Berlin, Stuttgart; 2000

44. Gauger T, Anshelm F, Schuster H, Draaijers GPJ, Bleeker A, Erisman JW, Vermeulen AT, Nagel HD: Mapping of ecosystem specific long-term trends in deposition loads and concentrations of air pollutants in Germany and their comparison with Critical Loads and Critical Levels. Part 1: Deposition Loads 19901999. Research Project 29942210 on behalf of Federal Environmental Agency. Final Report. Berlin, Stuttgart; 2002.

45. Gauger T, Anshelm F: Mapping of ecosystem specific long-term trends in deposition loads and concentrations of air pollutants in Germany and their comparison with Critical Loads and Critical Levels. Part 2: Mapping Critical Levels exceedances. Research Project 29942210 on behalf of Federal Environmental Agency. Final Report. Berlin, Stuttgart; 2002.

46. Gauger T, Haenel H-D, Rösemann C, Nagel H-D, Becker R, Kraft P, Schlutow A, Schütze G, Weigelt-Kirchner R, Anshelm F: Nationale Umsetzung der EU-NECRichtlinie/CAFE-Strategie und UNECE Luftreinhaltekonvention. Teil 2: Wirkungen und Risiokoabschätzungen, Critical Loads, Biodiversität, Dynamische Modellierung, Critical Levels Überschreitungen, Materialkorrosion. UBA-Texte 38/08. Dessau, 2008

47. Simpson D, Fagerli H, Hellsten S, Knulst JC, Westling O: Comparison of modelled and monitored deposition fluxes of sulphur and nitrogen to ICP-forest sites in Europe. Biogeosci 2006, 3:337-355.

48. Schaub M: Future monitoring and research needs for forest ecosystems in a changing environment: an introduction. iForest, 2009 2:54-55.

49. Schröder W, Pesch R: Long-Term monitoring of the metal accumulation in forests measured by use of the moss technique. Europ J Forest Res 2009, 129:475-488.

50. Ihle P (Hg): Atmosphärische Deposition in Deutschland. Teubner, Stuttgart, 2001:1-240.

51. Klockow $D$ et al.: Verfahren zur Erfassung der nassen und trockenen Deposition. In: Umweltschutz - eine internationale Aufgabe. Prag, 13.15.3.1985. Symposium und begleitende Ausstellung des Landes NordrheinWestfalen in Zusammenarbeit mit dem Nationalrat der CSSR: 7.1-7.32. Düsseldorf; 1985

52. Wilsnack $D$ : Vergleichbarkeit internationaler Luftqualitätsdaten im Untersuchungsgebiet. In Optimierung emissionsmindernder Maßnahmen bei gleichzeitiger Kontrolle der Aziditäts- und Luftschadstoffentwicklung für die Grenzregionen des Freistaates Sachsen. Hrsg. Sächsisches Landesamt für Umwelt und Geologie. Dresden; 2000:8-14.

53. Aas W, Alleman LY, Bieber E, Gladtke D, Houdret J-L, Karlssone V, Moniesf C: Comparison of methods for measuring atmospheric deposition of lead, cadmium, arsenic and nickel. J Environ Monit 2009, 11: 1276-1283.

54. Harmens H, Mills G, Hayes F, Jones L, Norris D, Cooper D and the participants of the ICP Vegetation: Air pollution and vegetation - ICP Vegetation annual report 2008/2009. Programme Coordination Centre for the ICP Vegetation, Centre for Ecology and Hydrology, Environment Centre Wales, Bangor, UK; 2009.

55. Holy M, Leblond S, Pesch R, Schroeder W: Assessing spatial patterns of metal bioaccumulation in French mosses by means of an exposure index. Environ Sci Pollut Res 2009, 16:499-507.

56. Schröder W, Englert C, Pesch R, Zechmeister H G, Thöni L, Suchara I, Maňkovská B, Jeran Z, Harmens H, Grodzinska K, Alber R:

Metallakkumulation in Moosen: Standörtliche und regionale Randbedingungen des Biomonitoring von Luftverunreinigungen. Umweltwiss Schadst Forsch 2008, 20:120-132.

57. Schröder W, Englert C, Pesch R, Zechmeister H G, Thöni L, Suchara I, Maňkovská B, Jeran Z, Harmens H, Grodzinska K, Alber R: Metal accumulation in mosses across national boundaries: uncovering and ranking causes of spatial variation. Environ Pollut 2008, 151:377-388.

58. Hellmann H: Definition der Hintergrund- bzw. Background-Konzentration - eine Übersicht. Acta Hydrochim Hydrobiol 2001, 29:391-398.

59. Brown DH: Uptake of mineral elements and their use in pollution monitoring. In The experimental biology of bryophytes. Edited by Dyer AF, Ducket JG. Academic Press, London, 1984:55-62

doi:10.1186/2190-4715-23-19

Cite this article as: Schröder W, et al: Mapping atmospheric depositions of cadmium and lead in Germany based on EMEP deposition data and the European Moss Survey 2005. Environmental Sciences Europe 2011, 23:19. 